AJChE 2006, Vol. 6, No. 2, 82-90

\title{
Base Case Simulation of a Semi-Batch Emulsion Copolymerization Process: Application to Styrene/Butadiene System
}

\author{
Iwan Harsono \\ Herman Hindarso \\ Nani Indraswati \\ Process System Engineering Laboratory \\ Department of Chemical Engineering \\ Widya Mandala Surabaya Catholic University \\ Kalijudan 37, Surabaya, INDONESIA 60114 \\ Email:iwanh626@mail.wima.ac.id
}

\begin{abstract}
It has been long recognized that emulsion polymerization is a complex heterogeneous process involving transport of monomers and other species and free radicals between aqueous phase and organic phases. Though there are a number of models available in the literature, most of them deal only with specific aspects in emulsion polymerization and are far from being general. To simulate this complicated process and to achieve an adequate level of understanding, a Polymer Plus ${ }^{\hat{b}}$ software from Aspen Technology, Inc. has been used. The objective of this work is to illustrate the principle use of Polymers Plus ${ }^{\hat{}}$ and to simulate and analysis the free-radical seeded emulsion copolymerization of styrene-butadiene process model in a semi-batch reactor. The base case simulation can be used to gain process understanding by analyzing how process variables and operating conditions during the course of a semi-batch reactor affect product quality.
\end{abstract}

Keywords: Emulsion copolymerization, Polymers Plus ${ }^{\mathrm{a}}$, simulation, semibatch reactor, and styrene/ butadiene.

\section{INTRODUCTION}

Emulsion polymers, or latexes, are polymer submicron-size particles dispersed in water. Stabilization of the particles is achieved by surfactants that tend to concentrate at the polymer surface. In the 1930s, the first emulsion polymerized styrene-butadiene rubber (SBR) known as Buna S was prepared by I. G. Farbenindustrie in Germany. Emulsion polymerized styrene-butadiene rubber (SBR) is one of the most widely used polymer in the world today. It is an industrially important process for the production of polymers used as synthetic rubber materials, inks, coatings application such as paints and adhesives; nonwoven fabrics. SBR production technology is detailed by Blackley and Lovell (Blackley, 1997; Lovell, 1997). About 2.5 million tons (International Institute of Synthetic Rubber Producers (IISRP), 2004) of SBR are produced annually.

The polymerization is usually carried out using water as the dispersion medium. This makes emulsion polymerization less detrimental to the environment than other processes in which volatile organic liquids are used as a medium. In addition, emulsion polymerization offers distinct processing advantages for the production of polymers. Unlike in bulk or solution polymerization, the viscosity of the reaction 
mixture does not increase as dramatically as polymerization progresses. For this reason, the emulsion polymerization offers excellent heat transfer throughout the course of polymer particles. This process is always chosen when the polymer product is used in latex form. In emulsion polymerization, free-radical propagation reactions take place in particles isolated form each other by the intervening dispersion medium. This reduces termination rates, giving high polymerization rates, and simultaneously makes it possible to produce high molecular weight polymers.

Many polymerization processes are neither batch nor continuous, but rather intermediate between two. These processes may be termed semibatch or semicontinuous. A reaction may be done in a semibatch or semicontinuous mode, or in stages for countless reasons. Common examples include promoting more desirable reaction path; to achieve a desired morphology; to adjust or maintain stoichiometry; to obtain a desired chain length, composition, or sequence length distribution; to modify the polymer product in some way; and for control purpose.

Batch and semibatch processes are usually dedicated to the low volume production of high value products or specialty grades. In semibatch processes (also referred to as semi-continuous batch processes) only part of the total reaction formulation is introduced at the beginning of the reaction, the remainder is being added, according to a predetermined schedule, during the course of the emulsion polymerization. In a typical semibatch process employing in-situ seeding, the heated initial charge of different materials is used for the production of seed particles; the radicals generated from the initiator become surface active and enter micelles to form particles.

The strategy of seeded emulsion polymerization is widely utilized, not only in batch processes, but also in semibatch and continuous processes. In seeded polymerization, particle nucleation is separated from particle growth. Seeded emulsion polymerization has two major advantages. Firstly, further complication of nucleation kinetics is avoided due to the absence of particle nucleation. Secondly, physical properties (such as particle size distribution and particle concentration) of the preformed particles can be fixed in advance, which the particle size distribution will be narrow if the seed stage is short.

Once the particles specifications or the reaction temperature are met, monomers and other ingredients, such as emulsifier, water and initiators are continuously added to the reactor according to some feed policies until the end of the batch. The surfactant amount must be selected so that it is just sufficient to maintain the colloidal stability of the preformed latex particles, but not so high as to generate new particles. It should be almost instantaneously mixed up with reactor contents. The particles are grown to the desired physical properties. For all semi-batch processes, the rate at which monomer is introduced to the reaction is of crucial importance because it controls the availability of monomer for polymerization and hence the concentration of monomer in the particles. The popularity of this method is easily understood; it grants safe operation and good control of both reaction exothermic and characteristics of the product. This kind of semi-batch operation is also favored because it provides great flexibility.

\section{THEORY}

The rough understanding of how emulsion polymerization proceed had to wait until the late 1940 s, for the initial qualitative description of Harkins $(1945,1947)$, which was fleshed out in more quantitative terms by Smith and Ewart (1948). Although theory of emulsion polymerization has become much more intricate in the years since, the theory of Harkins, Smith and Ewart remains the best point of introduction and of departure. With recent advances in both emulsion kinetics and polymer characterization, more in-depth studies have been carried out on important aspects in emulsion polymerization and better understanding has been achieved. A good review on recent advances in emulsion kinetics is given by Gilbert (1995). Researchers have also expanded their work from homopolymerization to copolymerization and even terpolymerization. There is a large body of models in the literature that describes certain aspects of emulsion homo-/copolymerization in recentyears (Gloor 1996, Sayer 1997, 2001, Yabuki 2000, Gao 2002, Zeaiter 2002, Casella 2003, Ginsburger 2003, Alhamad 2005). 
It is generally accepted that any one of the mechanisms could be responsible for particle formation depending on the nature of the monomer and the amount of emulsifier used in the recipe. The processes that can be modeled using the Polymers Plus ${ }^{a}$ emulsion polymerization model are those that follow two common mechanisms for particle formation: micellar or homogeneous polymerization. Homogeneous polymerization should become progressively more important with increasing monomer solubility in water and decreasing amount of surfactants.

Usually, anionic emulsifiers and water-soluble persulfate initiators are used for particle nucleation by the micellar mechanism. Micellar nucleation is considered to be the primary mechanism for particle formation (Harkin 1945, Smith and Ewart 1948 ) in those emulsion polymerization systems for which the monomer is very sparingly soluble in water, and where the concentration of emulsifier is above the critical micelle concentration (CMC).

The monomers are dispersed in the form of droplets in the water by agitation. The droplets formed are stabilized by the emulsifier molecules which are absorbed on the droplet surface. In addition to the droplets, monomer is also found dissolved in the aqueous medium and solubilized inside the micelles. Similarly, the emulsifier is found in three locations: in the micelles, dissolved in the aqueous medium, and adsorbed on the monomer droplets. Since water soluble initiator is used, the initiator molecules will be mainly found dissolved in the water medium.

When a typical emulsion polymerization recipe is heated, the initiator dissociates in the aqueous medium and produces initiator radicals. Upon propagating with monomer in the water phase the initiator radicals form oligomeric radicals and enter the micelles, which are aggregates of emulsifier molecules inside which a small amount of monomer is entrapped. The capturing of a radical by micelle and reaction with the entrapped monomer signifies the formation of a particle from a micelle (Stage I: Nucleation). The time required for particle nucleation to be complete is also called the nucleation time or the nucleation period, and usually lasts 10-15 minutes in conventional polymerization systems.

As the propagation takes place in the preformed seed particles, a thermodynamic potential difference is created for the diffusion of the monomer from the monomer droplets into the growing particles. The propagation stage (Stage II: Growth) is also know as the growth stage of the reaction, and is responsible for the development of the properties of the emulsion polymer: molecular weight, composition, micro structure, etc. As the particles grow, some of the micelles disintegrate and cover to stabilize them. Therefore, the micelles are not only consumed in the formation of polymer particles, but also in the stabilization of growing polymeric particles. As the particles grow, the emulsifier molecules are continuously adsorbed onto or desorbed from the particles to maintain thermodynamic equilibrium. Since the growth reaction lasts about 10-12 hours, there is great potential for optimizing the reaction time by increasing temperature or by keeping the particles saturated with monomer. The propagation stage is considered complete when the monomer droplets are totally depleted.

Since the number of particles remains constant and the particles are saturated with monomer, this stage is marked by a constant rate of polymerization and could easily be observed on a conversion versus time plot. Operational conditions can be chosen in such way that there are no monomer droplets in the reaction mixture. The condition is initially in the last stage of emulsion polymerization, where no monomer droplets are present. Only latex particles and the aqueous phase are present during this period, with the majority of the remaining monomer being confined to the latex particles, although a small amount (depending on the aqueous-phase solubility of the monomer) will be dissolved in the continuous phase. In the specific case of styrene-butadiene, which is sparsely water-soluble (water solubility of styrene and butadiene are 0.07 and $0.8 \mathrm{~g} / \mathrm{L}$ ), almost all monomer in the particle phase. The water solubility defines the amount of monomer available in the water phase to propagate the oligomeric polymerization. Particles as well as micelles are equally effective in capturing radicals from the aqueous phase. A growing radical can escape from a particle and return to the aqueous medium to participate in an aqueous phase termination reaction or enter into another particle. Radical generated in the aqueous phase are absorbed by the micelles during micellar nucleation and by the particle during nucleation and subsequent growth. 
In the last stage (Stage III: Monomer Finishing), the monomer finishing stage, the reaction mixture consists of the monomer swollen polymer particles and the aqueous medium. Further polymerization of the monomer in the particles takes place. During last stage, the concentration of monomer dissolved in the aqueous phase falls rapidly, as does the concentration in the polymer particles.

The polymerization of different monomers together to form a copolymer offers one of the simplest methods of improving (or at least changing) polymer properties, and one that has almost limitless variants. Obviously, the introduction of a second monomer brings with it the need to take into account the effects differences in monomer reactivity on the composition of the copolymer formed and the variation of copolymer composition with conversion. For emulsion polymerizations, the situation more complex than bulk or solution polymerizations due to the heterogeneous nature of the reaction.

Many of the monomers are actually more useful when copolymerized. For example, the homopolymer of styrene is poor in its resistance to organic solvents and ability to undergo environmental degradation; these disadvantages, coupled with brittleness and low upper use temperatures, effectively disqualify it for a number of applications. Copolymerization is often an attempt to obtain properties intermediate between those of homopolymers. Simultaneous polymerization of two or more monomers is a powerful and commercially important process for creating polymers with tailor made properties.

\section{STYRENE/BUTADIENE SYSTEM}

The simulation was based on a detailed fundamental model (Broadhead 1985) of a reactor carrying out the free radical emulsion copolymerization of styrene and butadiene. This resulting latex system will be furthermore used for paper coating application. Nevertheless, this final application is not the main topic of this work. In this process, the seeded emulsion copolymerization of styrene and butadiene is carried out in a semi-batch reactor using ammonium persulfate (APS) as the water-soluble thermal intiator, sodium lauryl sulfate (SLS) as the anionic emulsifier and tertiary dodecyl mercaptane (TDM) as a chain transfer agent. A schematic representation of the reactor is shown in figure 1. It consists of a batch reactor with an initial batch charge at $20^{\circ} \mathrm{C}, 10$ bar $(300 \mathrm{~kg}$ styrene, $300 \mathrm{~kg}$ butadiene, $30 \mathrm{~kg}$ SLS, $7 \mathrm{~kg}$ APS, $30 \mathrm{~kg}$ TDM, $1000 \mathrm{~kg}$ water) and a continuous feed at $20^{\circ} \mathrm{C}, 10$ bar $(700 \mathrm{~kg}$ styrene, $700 \mathrm{~kg}$ butadiene, $100 \mathrm{~kg}$ SLS, $3 \mathrm{~kg}$ APS, $1000 \mathrm{~kg}$ water).

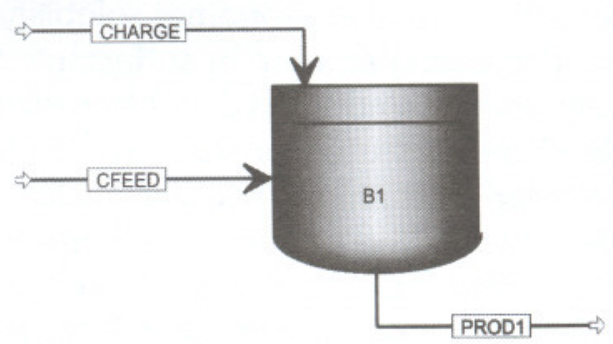

Figure 1. Reactor for Semibatch Emulsion Copolymerization of Styrene-Butadiene Rubber (CHARGE=initial charge stream, CFEED = continuous feed stream, and $P R O D 1=$ production stream)

The normal operating procedure for such a reactor starts with changing an initial recipe of differential material. Then, the reaction mixture is heated up to its reaction temperature profile. Once the reaction temperature is reached, monomers, initiator, emulsifier and water start being fed according to some feed policies until the end of the batch. The batch duration is assumed to be 10 hours for all case studies in this work. The Polymer Non-Random Two Liquid activity coefficient model (POLYNRTL) is used as the physical property method. The thermo physical properties of the monomers are obtained from the Aspen Plus pure component databank. The polymer physical properties are calculated using the van Krevelen method (Van Krevelen, 1990). The reaction type and reaction schemes as outlined in Table 1; rate constant for the kinetic scheme are obtained from Broadhead (1984).

The emulsion polymerization process is extremely complex and involves phenomena for which a complete theoretical understanding has not been reached. Following are the important model features and assumptions used in the emulsion polymerization model in Polymers Plus ${ }^{\mathrm{a}}$ : 
Table 1. The Reaction Type and Reaction Scheme

\begin{tabular}{|l|l|}
\hline Reaction type & Reaction scheme \\
\hline Initiator decomposition & $\begin{array}{l}\mathrm{I} \rightarrow \mathrm{n} \cdot \mathrm{R}^{+} \\
\text {Initiator } \rightarrow \text { Radicals }\end{array}$ \\
\hline Propagation & $\begin{array}{l}\mathrm{Pn}+\mathrm{M} \rightarrow \mathrm{Pn}+1 \\
\text { Active segment }+ \text { Adding monomer } \rightarrow \text { New active segment }\end{array}$ \\
\hline Chain transfer - monomer & $\begin{array}{l}\mathrm{Pn}+\mathrm{M} \rightarrow \mathrm{Dn}+\mathrm{P} 1 \\
\text { Active segment }+ \text { Transfer monomer } \rightarrow \text { Dead chain }+ \text { Active } \\
\text { segment }\end{array}$ \\
\hline Termination by combination & $\begin{array}{l}\text { Pn }+\mathrm{Pm} \rightarrow \mathrm{Dn}+\mathrm{m} \\
\text { Active segment }+ \text { Active segment } \rightarrow \text { Dead chain }\end{array}$ \\
\hline Chain transfer agent & $\begin{array}{l}\text { Pn }+\mathrm{A} \rightarrow \text { Dn }+\mathrm{R} \\
\text { Active segment }+ \text { Transfer agent } \rightarrow \text { Dead chain }+ \text { Radical }\end{array}$ \\
\hline
\end{tabular}

The reaction system is heterogeneous and formed by three phases: a monomer phase, an aqueous phase and a polymer phase. The reaction mixture is perfectly mixed.

Particles are formed by the micellar mechanisms. Due to the high surfactant concentration and sparsely water-soluble monomers used in this work, the second type of nucleation (i.e. homogenenous nucleation) is considered negligible.

No agglomeration or breakage of particles occurs.

No secondary nucleation occurs.

The polymerization follows the classical case 2 of the Smith-Ewart theory, which means that radical desorption is negligible and the average number of radicals per particle is equal to 0.5 .

All particles have the same average number of radicals and hence the same volumetric growth rate.

The particle size distribution is unimodal (monodisperse population of polymer particles), with moments of particle size distribution (PSD) sufficient to describe the PSD.

The concentration of the monomers in different phases is at the thermodynamic equilibrium.

There are no mass transfer limitations on the polymerization reactions.

Molecular weight is controlled by chain transfer reactions.

Pseudo-steady state hypothesis is used for the radical balance in the aqueous phase.

Terminal copolymerization model is used.

\section{RESULTS AND DISCUSSIONS}

The following figures show selected simulation results for a seeded emulsion copolymerization of styrene and butadiene in a semi-batch reactor. After a 1 hour seeding step as an initial charge, simulation were carried out with a 1 hour feeding time immediately after the end of seeding step with reaction temperature profile at $65^{\circ} \mathrm{C}$ from 0 to 0.5 hour, $70^{\circ} \mathrm{C}$ from 0.5 to 1 hour and $75^{\circ} \mathrm{C}$ from 1 to 10 hours (See Figures 2 and 3).

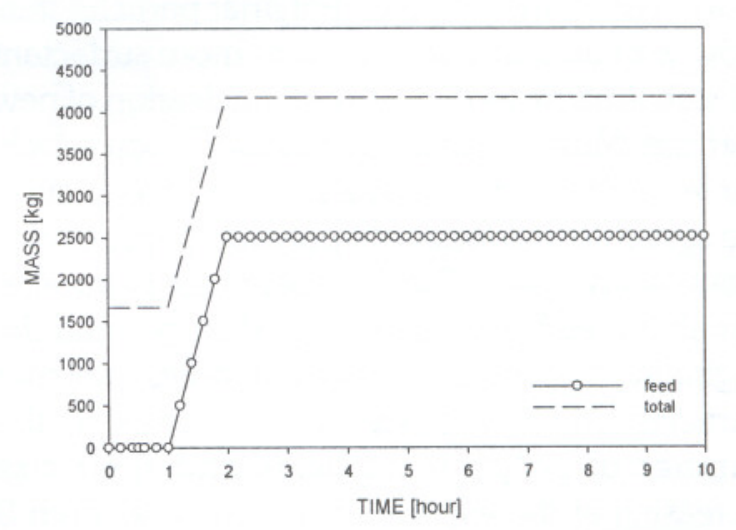

Figure 2. Total Mass and Continuous Feed Profile

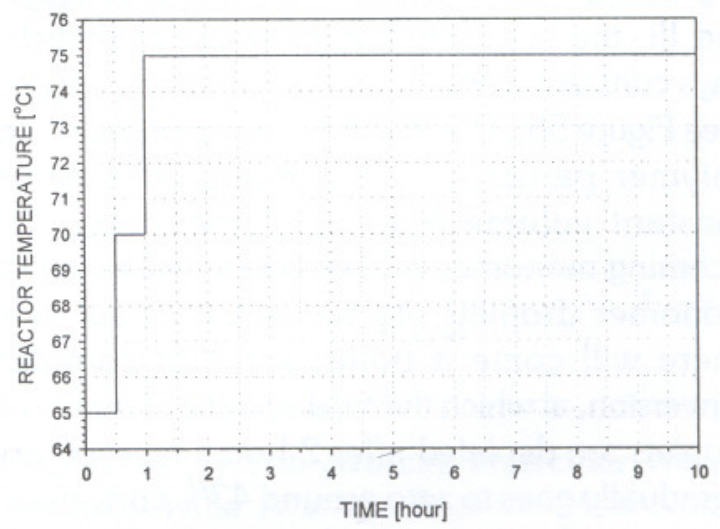

Figure 3. Reaction Temperature Profile 
The mass of monomers, polymer and other ingredients in the reactor is shown in figure 4. Although the flow rates of styrene and butadiene are equal, the figure shows that butadiene reacts fastor than sturono diso to its hishor roartivitu

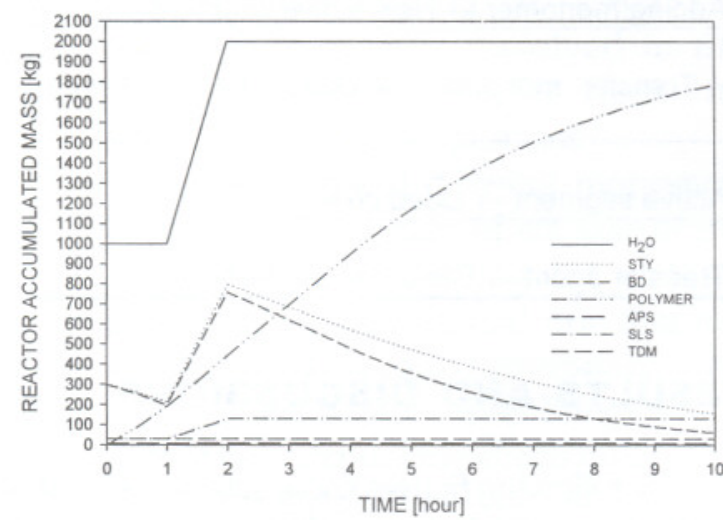

Figure 4. The Mass Monomer, Polymer, and Other Ingredients in the Reactor

Once nucleated (by whatever means), polymer particles adsorb more monomers to able to maintain monomer volume fractions, approximated by the equilibrium swelling of the monomers in the polymer. Polymer particles thus grow with time and this requires more surfactant for stabilization, as does further nucleation of new particles. Micelles are in this manner progressively depleted. When they have vanished completely, the system comprises only polymer particles and monomer droplets. The disappearance of micelles signals the end of what are called stage I and the beginning of stage II. In stage I, micelles, polymer particles and monomer droplets coexist, the number of polymer particles and their size increasing at the expense of the micelles from 0 to 0.021 hour (See Figure $5 \mathrm{a}$ ). The final number of particles is determined in this stage. In stage II and III, the concentration of polymer particles, stays constant while the polymerization proceeds (See Figure 5b). Polymerization occurs within the polymer particles, which maintain a nearly constant volume fraction of monomer. The incoming monomer is of course supplied by the monomer droplets via the aqueous solution. There will come a point, short of complete conversion, at which the total volume of monomer droplets are depleted after 2 hours reaction and it gradually goes to zero around $47 \%$ conversion, which marks the end of stage II. The only remaining monomer not in the polymer particles is in the aqueous phase. This marks the transition to stage III (See Figure 5c). As shown, the monomer droplets are completely depleted after 4 hours. As expected, the volume of the swollen
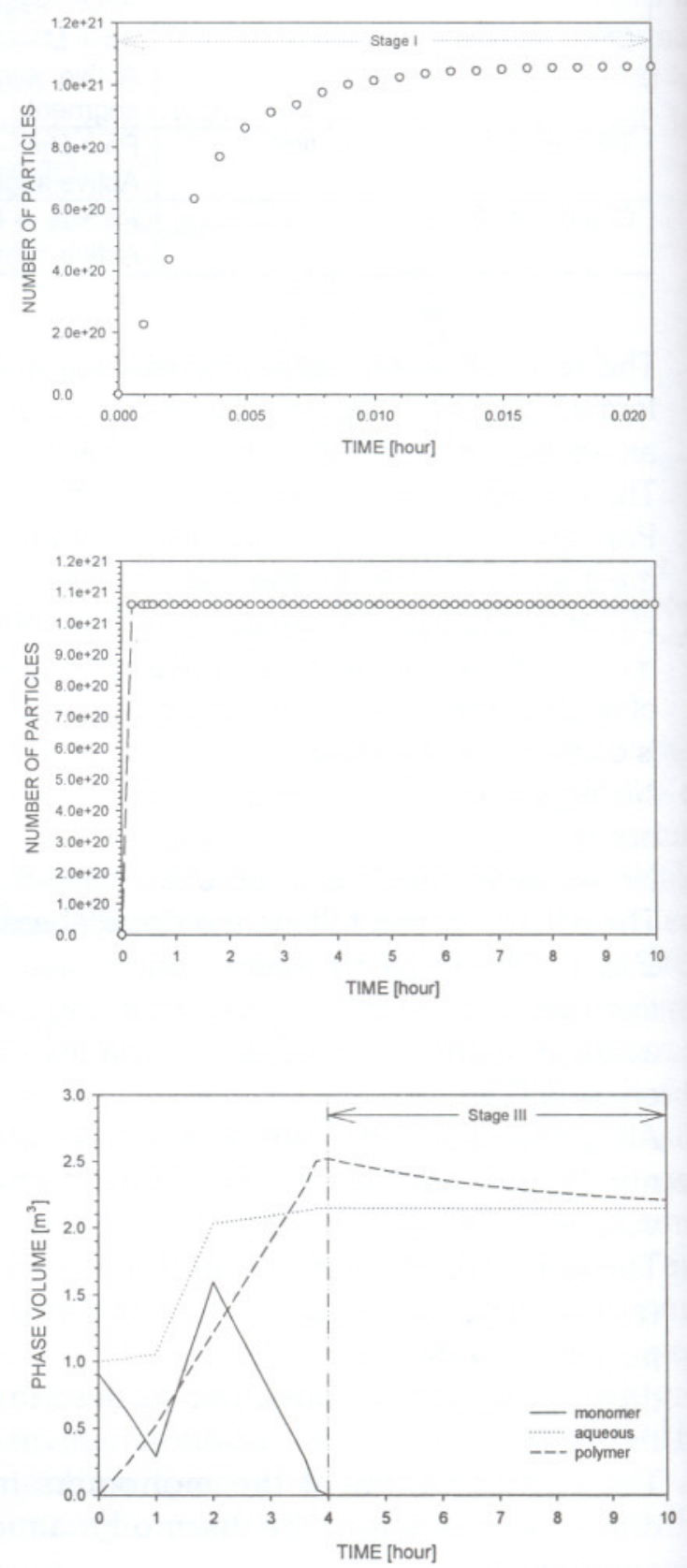

(c)

Figure 5. Three Stages of Emulsion Polymerization

Represented in terms of (a) the number of particles focusing on the nucleation stage, (b) the number of particles - focusing on the total reaction time and (c) total volume of the monomer, polymer and aqueous phase in the reactor. 
polymer phase decreases after the depletion of the monomer droplets. This last stage comprises consumption of the remaining monomer. The total volume of polymer particles increases before $47 \%$ conversion due to particle growth in stages I and II. It then decreases after $47 \%$ (in stage III) due to the density difference between monomer and polymer. The total volume of the aqueous phase remains nearly constant throughout the entire reaction. This division of an ideal emulsion polymerization into three intervals is helpful generalization implicit in the early theories.

As mentioned before, the starting point is case 2 kinetics and assumes that transfer is negligible. In the immediate termination of two radicals that occurs in this case, the two radicals are not equivalent; rather, one was a resident in the particle for some time and the other is a new arrival. The resident chains will be relatively long (and will have a geometric distribution), whereas the newly arrived chains will be short. The

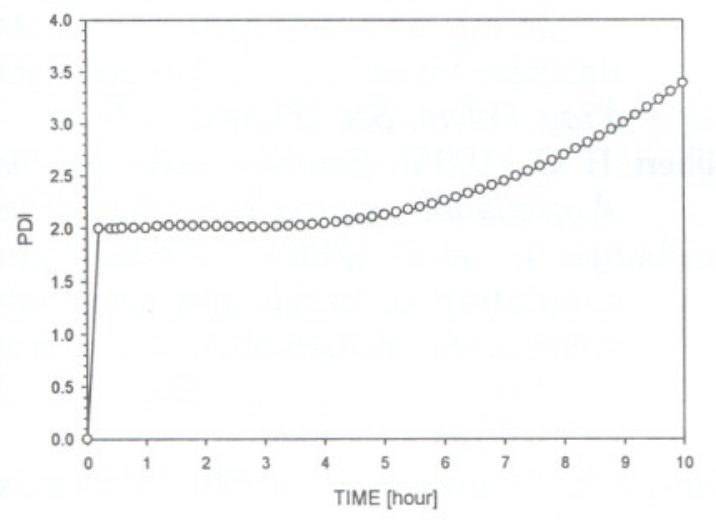

(a)

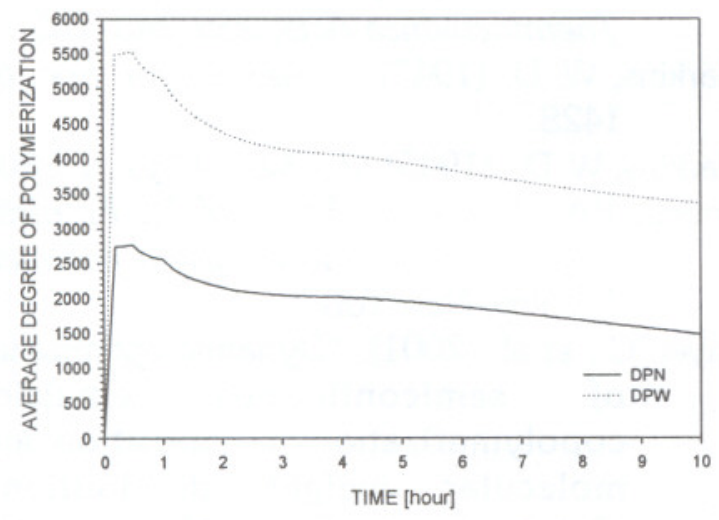

(b)

Figure 6. (a) The Number Average and Weight Average Degree of Polymerization, (b) the Polydispersity. resulting dead chain distribution will depend on the termination mechanism, as usual. If combination is dominant, each of the resident chain upon dying is increased by some small number of units, and the geometric distribution is roughly preserved, which will have a polydispersity (DPI) of approximately 2 (See

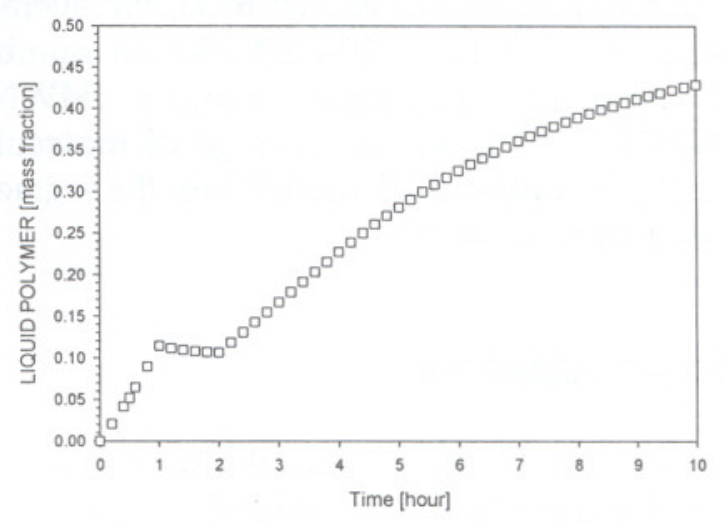

Figure 7. The Solid Content (Liquid Polymer) vs Time

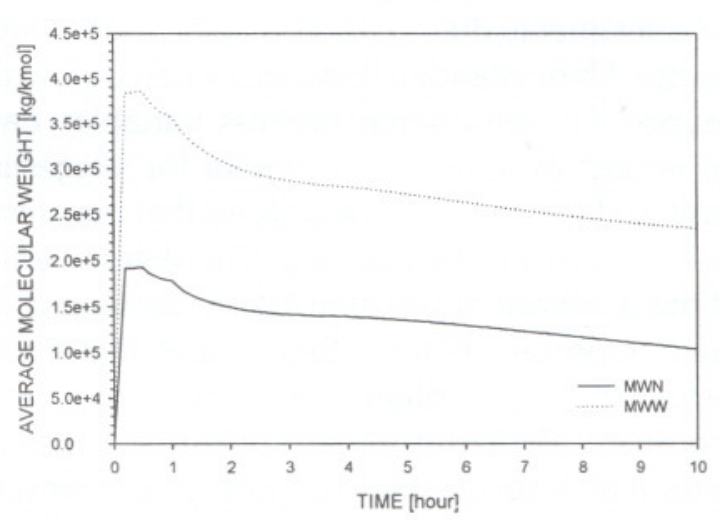

(a)

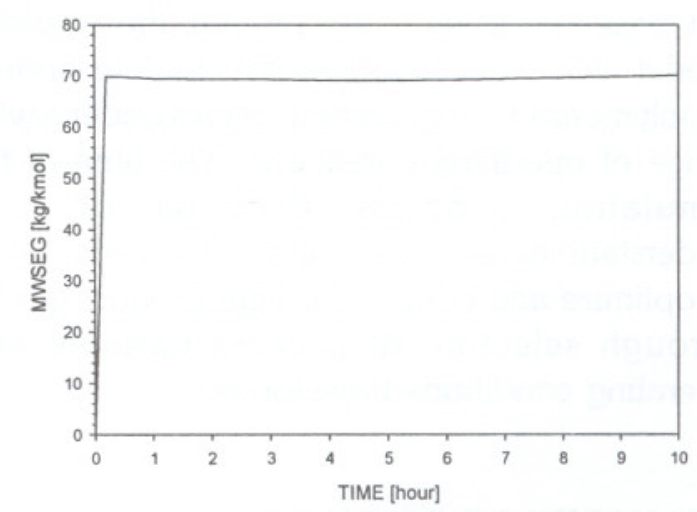

(b)

Figure 8. (a) The Average Molecular Weight and (b) the Average Segment Molecular Weight vs Time 
Figure 6a). The final product has average degree of polymerization of 1485.39022 for number average degree of polymerization (DPN) and 3353.34402 for weight average degree of polymerization DPW (See Figure 6b).

At the end of the reaction, the final polymer product has $43 \%$ solid content with overall conversion of $89 \%$ (See Figure 7), the average molecular weight of 104024.358 for number average of molecular weight (MWN), 234840.281 for weight average of molecular weight (MWW) and 70.1594983 for the segment (See Figure 8a and b).

\section{CONCLUSIONS}

The simulation provides the base case of a styrene-butadiene seeded emulsion copolymerization process in a semi-batch reactor, which can be used to study various process variables and operating conditions affect the product quality during the course of a semi-batch reactor. Understanding how and when during the course of a semi-batch process variables have an impact on quality is essential for suggesting modifications to operating policies that may result in improved product quality. The extensiveness of the database of this simulation package is the most important feature that makes this model distinct from most other models presented in the literature. The database contains physical/ chemical information for many monomers, initiators, emulsifiers and other emulsion polymerization ingredients. The richness of the database and its structure provide the emulsion model with great flexibility to simulate homo-/ copolymerization of different recipes under a wide range of operating conditions. The limit of the simulation progress depends on one understanding of the process. One could use it to optimize and control the final product quality through selection of process variable and operating conditions trajectories.

\section{ACKNOWLEDGMENTS}

Special thanks to Prof. I. L. Chien and Prof. C. S. Chern for valuable discussions and the Asian Development Bank for the funding part.

\section{REFERENCES}

Alhamad, B., Romagnoli, J. A., Gomes, V. G. (2005). "Advanced modeling and optimal operating strategy in emulsion copolymerization: Application to styrene/ MMA system," Chem. Eng. Sci., 60, 2795-2813.

Blackley, D.C. (1997). Polymer lattices: Science and technology, Chapman \& Hall, London.

Broadhead, T. O., Hamielec, A. E., MacGregor, J. F. (1985). "Dynamic modeling of the batch, semi-batch and continuous production of styrene/butadiene copolymers by emulsion polymerization," Makromolekulare Chemie. Suppl., 10/11, 105-128.

Casella, E. L., et al. (2003). "Mathematical modeling of batch emulsion copolymerization processes," Polymer Reaction Engineering, 11, 869-910.

Gao, J., Penlidis, A. (2002). "Mathematical modeling and computer simulator/ database for emulsion polymerizations," Prog. Polym. Sci., 27, 403-535.

Gilbert, R. G. (1995). Emulsion polymerization. A mechanism approach. Academic Press.

Ginsburger, E., et al. (2003). "Modelling and simulation of batch and semi-batch emulsion copolymerization of styrene and butyl acrylate," Chem. Eng. Sci., 58, 4493-4514.

Gloor, P. E., Warner, R. J. (1996). "Developing feed policies to maximize productivity in emulsion polymerization processes," Thermochimica Acta, 289, 243-265.

Harkins, W. D. (1947). J. Am. Chem. Soc. 69 , 1428.

Harkins, W. D., (1945). J. Chem. Phys., 13, 301.

Lovell, P.A., El-Aaser, M.S. (1997). Emulsion polymerization and emulsion polymers, J. Wiley, New York.

Sayer, C., et al. (2001). "Dynamic optimization of semicontinuous emulsion copolymerization: composition and molecular weight distribution," Computers and Chemical Engineering, 25, 839-849.

Sayer, C., Lima, E. L., Pinto, J. C. (1997). "Dynamic modeling of SBR emulsion polymerization reactors refrigerated by 
thermosyphons, Chem. Eng. Sci., 52, 341-356.

Smith, W. V., Ewart, R. H. (1948). J. Chem. Phys. 16, 592.

Van Krevelen, D. W. (1990). Properties of polymers, $3^{\text {rd }}$ ed., Elsevier, Amsterdam.

Yabuki, Y., Nagasawa, T., MacGregor, J. F. (2000). "An industrial experience with product quality control in semi-batch processes," Computers \& Chemical Engineering, 24, 585-590.

Zeaiter, J., Romagnoli, J. A., Barton, G. W., Gomes, V. G., Hawkett, B. S., Gilbert, R. G. (2002). "Operation of semi-batch emulsion polymerization reactors: modeling, validation and effect of operating conditions," Chem. Eng. Sci., 57, 2955-2969. 